\title{
Speed-power based training in the elderly and its potential for daily movement function enhancement
}

\author{
Nejc Šarabon (1,2,3), Darjan Smajla (1,2), Žiga Kozinc (1,4), Helmut Kern $(5,6)$ \\ (1) University of Primorska, Faculty of Health Sciences, Izola, Slovenia; (2) Innorenew CoE, \\ Izola, Slovenia; (3) S2P, Science to practice, Laboratory for Motor Control and Motor \\ Behaviour, Ljubljana, Slovenia; (4) University of Primorska, Andrej Marušič Institire, Koper, \\ Slovenia; (5) Ludwig Boltzmann Institute of Electrical Stimulation and Physical Rehabilitation, \\ Vienna, Austria; (6) Institute of Physical Medicine and Rehabilitation, Prim. Dr. H Kern GmbH, \\ Amstetten, Austria
}

This article is distributed under the terms of the Creative Commons Attribution Noncommercial License (CC BY-NC 4.0) which permits any noncommercial use, distribution, and reproduction in any medium, provided the original author(s) and source are credited.

\begin{abstract}
Resistance exercise is widely recommended strategy to improve functional ability and quality of life of the elderly. Novel resistance exercises approaches, such as flywheel exercise have recently emerged as superior alternatives to traditional methods. Eccentic exercise methods are well documented to elicit different and often the more pronounced effect on physical ability and function of the elderly. By reviewing the relevant scientific literature, we found that only limited studies have investigated the effects of flywheel exercise in the elderly, however, the results are very promising. Thus, more research is desired to explore the effect of flywheel exercise type in elderly individuals.
\end{abstract}

Key Words: older adults, seniors, strength, power, training.

Eur J Transl Myol 30 (1): xx1-xx4, 2020

With continous ageing of the world's populations it is important to investigate methods which may facilitate the physical ability and functional capacity of an older individual. One of the most detrimental changes, associated with ageing, is a $1-2 \%$ loss of muscle mass per year. This loss is assocociated with a $1-5 \%$ decrease in muscle strength and power, ${ }^{1}$ impaired balance, ${ }^{2}$ kineasthesia, ${ }^{3}$ and consequent decline in the quality of daily functional activites. Thus, preserving muscle mass and muscle activation shoud be one of the main approaches for maintaining daily function ability and independence in old age. One of the effective methods for prevention of changes caused by aging is resistance training. With appropriate training methods, older people can slow down sarcopenia, increase the cross-sectional area of fast-twitch muscle fibers, improve mucle strenght which positively influence daily function ability. Until now, traditional resitance exercise with high-loads (hypertrophy) methods and methods for improving muscle activation have been recommended to counteract negative effects of aging. ${ }^{4,5}$ Literature suggests that the ability to produce power declines faster than strength, especially in the proximal muscles of the lower limbs compared to the upper limb. ${ }^{6}$ This negatively affects postural control, movement function and increases the risk of falls, ${ }^{7}$ which suggests that proper loading of the proximal body parts in the elderly may be very effective. Recent studies have shown that resistance training that includes performing repetitions with maximal velocity results in higher performance gains and greater associations with the physical and functional abilities of the elderly compared to strength training alone. ${ }^{8}$

Previous resistance training studies used different types of loading and equipment: body-weight training, ${ }^{9}$ training with elastics, ${ }^{10}$ and different weight devices. ${ }^{11}$ In general, all training methods have a positive effect on the physical abilities of the elderly. However, exercise training that is designed solely to increase the maximum strength, may not elicit significant improvements in balance or functional abilities. Traditional resistance exercise methods are limited by the load which an individual is capable to overcome in the concentric phase of muscle contraction and because of that they have less potential for developing rapid strength and power. During daily tasks, the elderly are constantly confronted with eccentric contractions, i.e., the muscular contractions during which the active muscle group is lengthening/braking (walking down the stairs, sitting down and getting up, balance maintenance, etc.) The load exerted by the active muscle group during breaking to the 


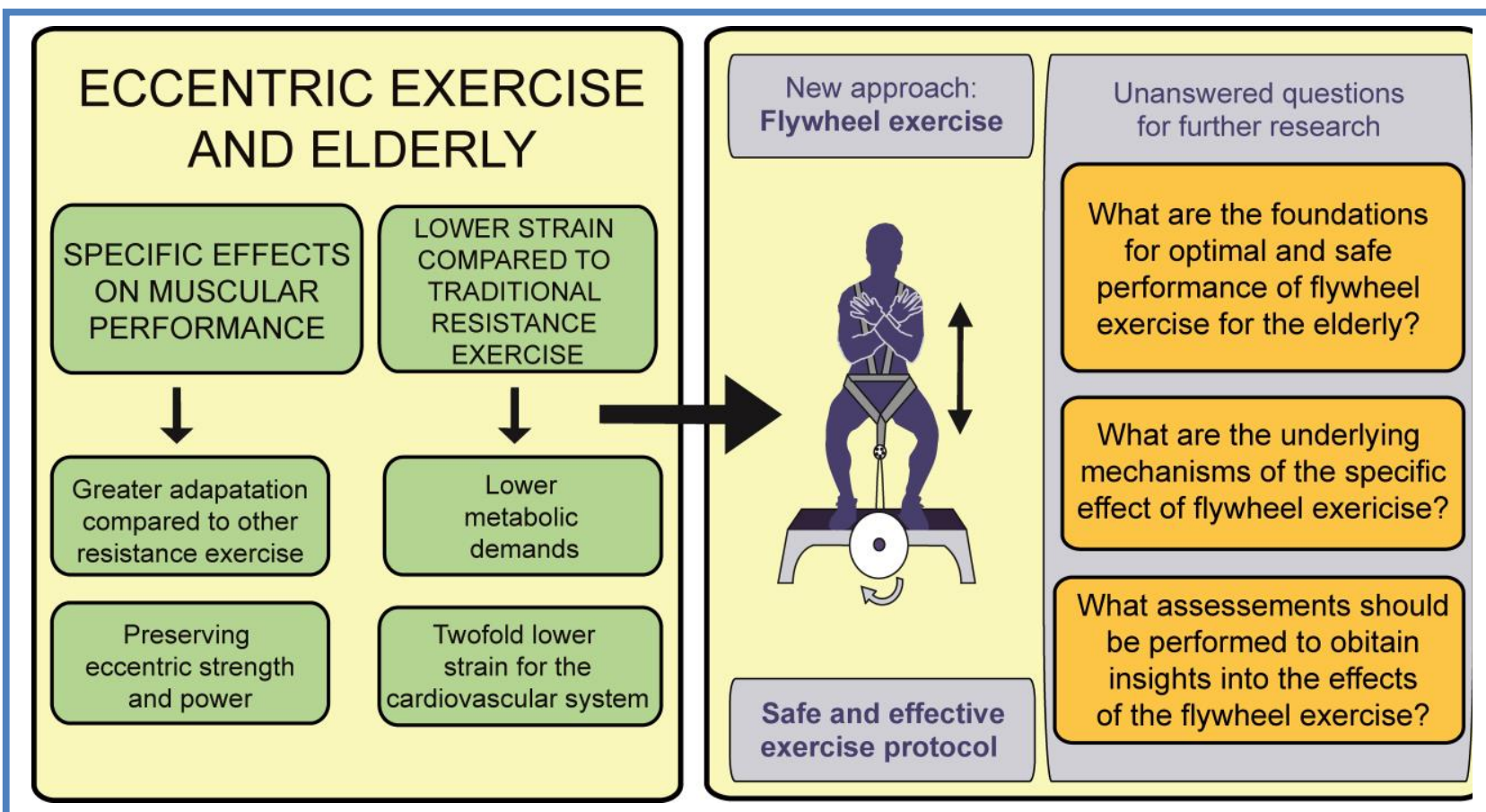

Fig 1. Eccentric exercise and elderly

external load represents a different and potentially better stimulus for neuromuscular adaptation in the elderly (Figure 1). Exercise that enables high power development and includes eccentric contraction can be performed on flywheel devices. Flywheel exercise allow for resistance training at different speeds in the concentric and eccentric mode of muscle contraction, therefore it has good potential to affect the daily movement function in the elderly. However, there is a lack of evidence regarding the intensity of flywheel exercise that would allow systematic progression and personalization of exercise protocols for maximizing training effects and considering terms of safety for the elderly population.

\section{Materials and Methods}

We systematically reviewed flywheel intervention studies on the elderly population that included both muscle power and at least one index of functional performance as an outcome measure. Several international scientific databases were searched for peerreviewed articles with the following keywords (and combinations): elderly, flywheel, iso-intertial, eccentric, exercise. The studies were critically reviewed and qualitatively compared.

\section{Results and Discussion}

Although the general concept of eccentric exercise is well known, there are still many open questions regarding the implementation of flywheel exercise for the elderly. There are only three studies in the literature that have used flywheel devices to train healthy elderly people. In one study involving 24 older individuals, a group that trained knee extensors for 12 weeks using a flywheel device had better long-term results compared to a group that trained using free weights. ${ }^{12}$ In the flywheel exercise group, there was a significant increase in the peak isokinetic power of knee extensors, an increase in the gastrocnemius tendon stiffness, and an improvement in postural balance compared to the other group. Another study reported an increase in knee extensor strength, a decrease in LDL cholesterol, and improved quality of life in the group of elderly who trained on flywheel device, while the group that performed aerobic interval training improved only in terms of aerobic capacity and blood pressure. ${ }^{13}$ The only study on the elderly that used an flywheel squatting device reported significant positive effects of flywheel exercise on mobility, power, and balance, although it was compared only with a nonexercise control group. ${ }^{14}$ In summary, flywheel studies in healthy elderly people reported similar or greater increases in muscle power. This may confirm flywheel exercise as a potentially superior method for prevention of sarcopenia compared to other resistance exercise methods. Moreover, implementation of training that includes eccentric overload improves postural balance of the elderly. ${ }^{12,15}$ Two studies, indluding elderly participants with chronic non-communicable diseases also verifed the potential of flywheel exercise for the elderly (increase in muscle mass, strength and power of the injured side, improvement in postural balance, gait characteristics and daily function). ${ }^{16,17}$ Although the number of studies that investigated the effects flywheel training for the elderly is very small, their findings report important muscular, functional and clinical adaptations. 
With flywheel training, we can safely achieve heterogenic and economic exercise effects, which in practice prove to be important characteristics of exercise in the elderly. To date studies have not provide answers about appropriate intensity, progression, optimal volume, tempo, and exercise adjustment for the elderly that would maximize the effects of exercise. In one study, the intensity was systematically adjusted and the final result was a $28 \%$ increase in the maximum power of the knee extensors. ${ }^{12}$ However, the findings of this study are limited to localized effects, since the exercise protocol contained flywheel knee extension in a seated position. In the study where they compared aerobic interval training with flywheel training in the elderly, the authors did not report the intensity of the flywheel training, ${ }^{13}$ making the comparison between the studies impossible. In the only study in which the elderly performed squats on flyheel device, ${ }^{14}$ the authors reported difficulties in determining exercise intensity. This can explain the absence of muscle adaptations. At the same time, the lack of effects of flyheel exercise on balance was though to be the consequence of short duration of the exercise intervention ( 6 weeks). Based on the literature, there is a lack of evidence on the field of flywheel exercise loading and its prescprition. More research is needed to provide information on approprate personalization and planning of the training process in order to maximize the effect of the exercise and ensure safety. To date, studies have not provided much insight into the relevant chronic effects of systematic and adapted flywheel exercise for the elderly. Exercise protocols and load intensities within these studies have not been designed in view of scientific evidence and necessary adjustments for the elderly population. Moreover, evidence of underlying exercise mechanisms and measurement procedures for their verification of are largely unexplored. Flywheel training on squatting device imitates daily activities, such as sitting down and getting up, which are crucial for the elderly as they are strongly associated with the mobility and daily function. With appropriate and systematic loading, flywheel exercise may represent a superior stimulus for improvements in proprioception and kinaesthetic sense, compared to weight training. All to all, this training modality offers innovative solutions for individualized training progression (load, tempo, postural stability and is more economical for the elderly (parallel strength and balance training). In view of the outlined paucity of evidence, our plan is to obtain a better insight into the potential that this type of resistance training has for changing force-velocity-power profile in elderly. This would complement previously used testing approaches we used in past interventional studies in elderly. ${ }^{18}$ Namely, improving velocity dominance in the force-velocity profile seems to have significant functional relevance (balance control, gait initiation, change of direction; and prevention of falls as a result). ${ }^{19}$ Results on initial methodological considerations and protocols development will be presented.

\section{List of acronyms}

None

\section{Acknowledgements}

The review was performed in the preparatory phase of the planned intervention studies to be performed in the project "2014 - 2020 INTERREG V-A Slovakia Austria: Project - Centre of Active Ageing - Centrum of competences for physical activity in everyday life; prevention and health promotion of the elderly."

\section{Authors contributions}

NŠ and HK contributed to review problem definition and review conception. NŠ, DS and $\breve{Z} \mathrm{~K}$ did the literature search, papers acquisition, selection and information extraction, as well as drafted the paper.

\section{Funding}

Project "2014 - 2020 INTERREG V-A Slovakia Austria: Project - Centre of Active Ageing - Centrum of competences for physical activity in everyday life; prevention and health promotion of the elderly."

\section{Conflict of Interest}

The authors declare they have no financial, personal, or other conflicts of interest.

\section{Ethical Publication Statement}

We confirm that we have read the Journal's position on issues involved in ethical publication and affirm that this report is consistent with those guidelines.

\section{Corresponding Author}

Nejc Šarabon, University of Primorska, Faculty of Health Sciences, Polje 42, 6310 Izola.

ORCID iD: 0000-0003-0747-3735

Email: nejc.sarabon@fvz.upr.si

E-mail of co-authors

Darjan Smajla: darjan.smajla@fvz.upr.si

ORCID iD: 0000-0001-5441-8988

Žiga Kozinc: ziga.kozinc@fvz.upr.si

ORCID iD: 0000-0003-3555-8680

Helmut Kern: helmut@kern-reha.at

ORCID iD: 0000-0001-9661-8814

\section{References}

1. Deschenes M. Effects of Aging on Muscle Fibre Type and Size. Sport Med 2004;34:809-24.

2. Sohn J, Kim S. Falls study: Proprioception, postural stability, and slips. Biomed Mater Eng 2015;26:S693-703.

3. Ribeiro F, Oliveira J. Aging effects on joint proprioception: the role of physical activity in proprioception preservation. Eur Rev Aging Phys Act 2007;4:71-6.

4. Zampieri S, Mosole S, Löfler S, Löfler S, Fruhmann $\mathrm{H}$, Fruhmann H, et al. Physical exercise in Aging: Nine weeks of leg press or electrical stimulation 


\section{Speed-power based training in the elderly}

Eur J Transl Myol 30 (1): xx1-xx4, 2020

training in 70 years old sedentary elderly people. Eur J Trans1 Myol 2015;25:237. doi:10.4081/ejtm. 2015.5374

5. Chodzko-Zajko WJ, Proctor DN, Fiatarone Singh MA, et al. Exercise and physical activity for older adults. Med Sci Sports Exerc 2009;41:1510-30. doi:10.1249/ MSS.0b013e3181a0c95c

6. Häkkinen K. Ageing and Neuromuscular Adaptation to Strength Training. In: Komi P, editor. Strenght and power in sport The encyclopedia of sports medicine. Oxford: Blackwell scientific publishing; 2003. pp. 409-25.

7. Ponce-Bravo H, Ponce C, Feriche B, Padial P. Influence of two different exercise programs on physical fitness and cognitive performance in active older adults: Functional resistance-band exercises vs. recreational oriented exercises. J Sport Sci Med 2015;14:716-22.

8. Byrne C, Faure C, Keene DJ, Lamb SE. Ageing, Muscle Power and Physical Function: A Systematic Review and Implications for Pragmatic Training Interventions. Sport Med 2016;46:1311-32. doi:10.1007/s40279-016-0489-x

9. Ramírez-Campillo R, Castillo A, de la Fuente CI, et al. High-speed resistance training is more effective than low-speed resistance training to increase functional capacity and muscle performance in older women. Exp Gerontol 2014;58:51-7. doi:10. 1016/j.exger.2014.07.001

10. Sturnieks DL, St George R, Lord SR. Balance disorders in the elderly. Neurophysiol Clin 2008;38:467-78. doi:10.1016/j.neucli.2008.09.001

11. Henwood TR, Riek S, Taaffe DR. Strength versus muscle power-specific resistance training in community-dwelling older adults. J Gerontol - Ser A Biol Sci Med Sci 2008;63: 83-91. doi:10.1093/gerona/63.1.83

12. Onambélé GL, Maganaris CN, Mian OS, et al. Neuromuscular and balance responses to flywheel inertial versus weight training in older persons. $\mathrm{J}$ Biomech 2008;41:3133-8. doi:10.1016/j.jbiomech.
2008.09.004

13. Bruseghini P, Calabria E, Tam E, et al. Effects of eight weeks of aerobic interval training and of isoinertial resistance training on risk factors of cardiometabolic diseases and exercise capacity in healthy elderly subjects. Oncotarget 2015;6:1699817015.

14. Sañudo B, González-Navarrete Á, Álvarez-Barbosa $F$, et al. Effect of flywheel resistance training on balance performance in older adults. A randomized controlled trial. J Sport Sci Med 2019;18:344-50.

15. Reeves ND, Maganaris CN, Longo S, Narici MV. Differential adaptations to eccentric versus conventional resistance training in older humans. Exp Physiol 2009;94:825-33. doi:10.1113/exp physiol.2009.046599

16. Zaleski AL, Taylor BA, Panza GA, Wu Y, Pescatello LS, Thompson PD, et al. Coming of age: considerations in the prescription of exercise for older adults. Methodist Debakey Cardiovasc J 2016;12:98-104.

17. Clark DJ, Patten C. Eccentric versus concentric resistance training to enhance neuromuscular activation and walking speed following stroke. Neurorehabil Neural Repair 2013;27:335-44. doi:10.1177/1545968312469833

18. Sarabon N, Löfler S, Hosszu G, Hofer C. Mobility test protocols for the elderly: a methodological note. Eur J Transl Myol 2015;25: 253. doi:10.4081/ejtm.2015.5385

19. Gianoudis J, Bailey C, Ebling P, et al. Effects of a targeted multimodal exercise program incorporating high-speed power training on falls and fracture risk factors in older adults: a community-based randomized controlled trial. J Bone Min Res 2014;29:182-91.

Submissions: Febraury 12, 2020

Revision received: Febraury 18, 2020

Acceptance: Febraury 21, 2020 